\title{
Actitudes hacia las matemáticas en estudiantes de Honduras
}

Daniel Enrique Pineda Ramírez ${ }^{1}$ Sayda Patricia Palma Martínez ${ }^{2}$ Carlos Roberto Pérez Dubón ${ }^{3}$

\section{Información de artículo:}

Recibido: 30/03/2021

Aprobado: 30/05/2021

\section{Palabras claves:}

Actitudes, dominio afectivo, género, matemáticas, COVID-19

\section{Keywords:}

Attitudes, affective domain, gender, mathematics, COVID-19

\section{Resumen:}

Esta investigación ha analizado las actitudes hacia las matemáticas de estudiantes de Educación Básica y Secundaria de Honduras. Se trata de una investigación cuantitativa sustentada en un diseño Ex Post Facto, donde se suministró una escala de actitud hacia las matemáticas a 597 estudiantes. Los principales resultados muestran diferencias estadísticamente significativas entre las variables demográficas en relación con el factor percepción de incompetencia matemática. Además, al analizar cada uno de los factores se ha encontrado de forma general que los estudiantes tienen una actitud positiva hacia las matemáticas en los factores percepción de incompetencia matemática, percepción de utilidad y autoconcepto matemático, al contrario, con el factor gusto por las matemáticas en el cual se ha observado una actitud negativa al respecto. Se concluye que los estudiantes de zonas urbanas tienen mejores actitudes que los estudiantes de zona rural, esto se debe al acceso a los servicios y destreza tecnológica de los estudiantes urbanos en tiempos de COVI-19.

\section{Attitudes towards mathematics in Honduran students}

\section{Abstract:}

This research has analyzed the attitudes towards mathematics of students of Basic and Secondary Education in Honduras. This is a quantitative research based on an Ex Post Facto design, where a scale of attitude towards mathematics was provided to 597 students. The main results show statistically significant differences between the demographic variables in relation to the perception

\footnotetext{
${ }^{1}$ Licenciada en Matemáticas, Profesor de Matemáticas en el Instituto Tecnológico en Administración de Empresas, danikikeramirez@gmail.com, (ID) https://orcid.org/o0oo-0002-2915-3815

${ }^{2}$ Licenciada en Matemáticas, Profesora de Matemáticas en Instituto José Antonio Ochoa, patyo888@gmail.com, (1): https:// orcid.org/0000-0002-3950-0067

3 Ingeniero en Sistemas Computacionales. Profesor de la Universidad Tecnológica Centroamericana. crpdubon @gmail.com, (D): https://orcid.org/oooo-0002-2696-7741
} 
factor of mathematical incompetence. In addition, when analyzing each of the factors, it has been found in a general way that students have a positive attitude towards mathematics in the factors perception of mathematical incompetence, perception of usefulness and mathematical self-concept, on the contrary, with the factor taste for mathematics in which a negative attitude has been observed in this regard. It is concluded that students from urban areas have better attitudes than students from rural areas, this is due to access to services and technological skills of urban students in times of COVI-19.

\section{Introducción}

El presente estudio ha analizado las actitudes hacia las matemáticas en estudiantes de educación Básica y Secundaria en Honduras. Se muestra la relación entre las actitudes hacia las matemáticas y el proceso enseñanza-aprendizaje, en este sentido, se parte de la definición de actitud hacia las matemáticas, así como, los componentes relacionados y la relevancia que tienen dentro del proceso, sin embargo, no se puede hablar de actitudes hacia las matemáticas sin mencionar que estas se relacionan con la parte afectiva y cognitiva de los estudiantes dejando entre ver los componentes que se derivan. Por su parte, Martínez (2008) y Cantero et al. (2008) mencionan que las actitudes pueden manifestarse o expresarse mediante factores tales como ideas, percepciones, gustos, preferencias, opiniones, creencias, emociones, sentimientos comportamientos y tendencias a actuar.

Son varios los estudios sobre actitudes matemáticas que se han realizado al igual se encuentran estudios que proponen la elaboración de instrumentos para medir las actitudes hacia las matemáticas destacando entre ellos los de Aiken y Dreger (1961), Fennema y Sherman (1976), Flores y Auzmendi (2018), con el propósito de medir la "afectividad, agrado", "ansiedad, miedo", "valor y utilidad", "motivación" y "seguridad, confianza" hacia las matemáticas. En este sentido, esta investigación trata de medir las actitudes hacia las matemáticas mediante una escala tipo Likert que contempla cuatro factores los cuales son: percepción de la incompetencia matemática, gusto por las matemáticas, percepción de utilidad, autoconcepto matemático los cuales se estarán detallando más adelante (Palacios et al., 2014).

\section{Literatura}

La actitud hacia el proceso de enseñanza-aprendizaje de las matemáticas, forma parte imprescindible para el aprendizaje, esto debido que estas están relacionadas con las creencias del individuo y su formación, lo que impacta sobre sus logros y los productos de sus actividades (Morales et al., 2013). Según Nortes et al. (2017) el estudio de las actitudes constituye una herramienta analítica útil para evaluar la eficacia del proceso de aprendizaje y las potenciales innovaciones producidas en el mismo. Esto debería ser un incentivo para que los docentes del área realicen observaciones 
e investigaciones de las actitudes de sus estudiantes hacia la matemática, con el fin de tomar decisiones ayuden a mejorarlas y de esta manera se mejore el proceso de enseñanza - aprendizaje de las matemáticas.

La manifestación explícita o implícita de las actitudes se expresa en términos de factores tales como "ideas, percepciones, gustos, preferencias, opiniones, creencias, emociones, sentimientos, comportamientos y tendencias a actuar" (Martínez, 2008, p. 244). Por lo que pueden ser observables en el educando y a su vez trazan una línea que tiende a determinar un comportamiento hacia la clase y la forma de desenvolverse dentro y fuera de la misma. Para poder medir las actitudes se han desarrollado ciertos componentes o dimensiones como los mencionados por Morales et al. (2013) quien caracteriza las actitudes en tres componentes: el cognitivo, el afectivo y el conductual, sin embargo, Martínez, (2008) propone uno más que es el conativo o intencional.

- Componente Cognoscitivo (el conocer / el saber): "se expresa mediante percepciones, ideas, opiniones, concepciones y creencias” (Martínez, 2008, p. 244). La resolución de problemas y la elección de procedimientos que permitan obtener una respuesta de un ejercicio matemático, como la coherencia de las ideas y opiniones vertidas por el alumno dentro del aula de clase denotan el impacto cognoscitivo obtenido de las actitudes del estudiante.

- Componente Afectivo (la emoción / el sentir): "este componente se pone de manifiesto por medio de las emociones y los sentimientos de aceptación o de rechazo" (Martínez, 2008, p. 244). La aceptación o resiliencia a los contenidos matemáticos de la clase, las emociones que pueda sentir el estudiante cuando llega el momento de recibir la clase, el sentimiento de frustración o éxito al momento de realizar las tareas de matemáticas son las que denotan el componente afectivo del Estudiante hacia la clase de matemáticas.

- Componente Conativo o Intencional (la intención): “constituido por predisposiciones, predicciones, preferencias, tendencias o intenciones de actuar de una forma específica ante el objeto" (Martínez, 2008, p. 244). La predisposición del estudiante hacia la realización de actividades que involucren un esfuerzo extra por parte de este, la tendencia en realizar las tareas en un tiempo calendarizado o improvisado a último momento, las preferencias por realizar primero o al final las tareas de la clase de matemáticas por sobre las tareas de otras materias, determinan el componente conativo de las actitudes hacia las matemáticas

- Componente Comportamental (el comportamiento): es el componente característico de las matemáticas que puede determinarse únicamente mediante la observación del comportamiento del educando dentro del aula de clase (Martínez, 2008). 
Al momento de hablar de actitudes en matemáticas, cabe señalar la diferencia entre actitudes matemáticas y actitudes hacia la matemática, las primeras hacen referencia al componente cognitivo, puesto que están relacionadas con el actuar sobre la actividad matemática, en contraste, las segundas involucran el componente afectivo, el cual toma en cuenta valoraciones y el disfrute por las matemáticas (Palacios, Arias y Arias, 2014). De esta manera, Palacios et al. (2014), desarrollan la Escala de Actitudes hacia las Matemáticas (EAM). La EAM mide las actitudes hacia las matemáticas, es decir el componente afectivo, dividido en 4 factores estos son: percepción de la incompetencia matemática, gusto por las matemáticas, percepción de utilidad y autoconcepto matemático, a continuación, su descripción:

- Percepción de incompetencia matemática: está determinado por la seguridad del estudiante para adquirir y utilizar competencias matemáticas. Su medición se basa en el intervalo que va desde una seguridad definida, hasta una deficiencia en su seguridad sobre su competencia matemática (Fennema y Sherman, 1976).

- Gusto por las matemáticas: comprende el agrado por resolver problemas, conceptos matemáticos, simbología matemática y ejercicios de cálculo (Aiken, 1974) en el mismo sentido, Auzmendi (1992) y Nortes y Nortes (2017), menciona que tiene que ver con el gusto de realizar actividades o trabajos matemáticos.

- Percepción de utilidad: combinando ideas de Fennema y Sherman (1976), y Auzmendi (1992), este factor es referente al valor, creencias o percepciones que los estudiantes consideren sobre la utilidad que se les dan a las matemáticas en la vida cotidiana, como soporte de vocaciones o en su futuro ámbito laboral.

- Autoconcepto matemático: según Gómez-Chacón (1997) y Gómez-Chacón (2000), se basa en la autoimagen del estudiante sobre las matemáticas y cómo las perciben. Está vinculado con los ideales del aprendiz sobre las matemáticas, los cuales se ha desarrollado durante su proceso de enseñanza aprendizaje.

Con relación a estudios realizados anteriormente donde se ha evaluado las actitudes hacia las matemáticas comparando el género, y grado académico se han encontrado los siguientes resultados: En la investigación de Zarrazaga (2006), donde se analizó el agrado y temor por las matemáticas se detalló que los estudiantes poseen una actitud negativa hacia las matemáticas; al analizar las variables por género no encontró diferencias significativas en estas variables. Sin embargo, sí se encontraron diferencias significativas entre las actitudes hacia las matemáticas y grado académico teniendo un comportamiento inversamente proporcional, es decir a mayor el grado académico, la actitud hacia las matemáticas se volvía más negativa. Finalmente, en la investigación realizada por Muñoz y Mato (2008), donde se estudió el agrado y utilidad de las matemáticas, se encontró que en los estudiantes no había diferencias 
significativas entre el género y en el caso de del grado académico no hubo diferencias significativas entre primer curso y tercero, al contrario de segundo curso y cuarto donde se encontrar diferencias con una tendencia inversamente proporcional.

\section{Metodología de investigación}

\subsection{Finalidad y objetivos del estudio}

Esta investigación tiene por objetivo general determinar si los estudiantes tienen una actitud positiva o negativa en el proceso de enseñanza-aprendizaje de las matemáticas, tomando en consideración las variables género, edad, grado académico y zona de residencia de los estudiantes, por tales razones, se proponen los objetivos específicos siguientes: (1) Identificar si las actitudes hacia las matemáticas del estudiantado en la enseñanza-aprendizaje de las matemática es positiva o negativas; (2) Explicar la relación entre los factores actitudinales en la enseñanza de las matemáticas; (3) Comprobar si existen diferencias significativas entre género, edad, grado académico y zona de residencia en relación con las actitudes hacia las matemáticas del estudiantado.

\subsection{Metodología de la investigación}

El enfoque de esta investigación es cuantitativo. Este paradigma ha guiado el tratamiento de los datos a través de la categorización y descripción de las propiedades, características y perfiles de las personas, grupos, comunidades, procesos y objetos que se han sometidos a análisis (Hernández et al. 2014). El diseño de esta investigación es "Ex Post Facto" porque este tipo de investigación trata de descubrir fenómenos que ocurren en forma natural, pero miden diversas variables para analizar su posible efecto (Bisquerra et al, 2012).

\subsection{Descripción de los participantes}

El estudio se llevó a cabo con una muestra de 597 estudiantes de diferentes instituciones hondureñas.

\begin{tabular}{|l|c|c|c|}
\hline \multicolumn{4}{|c|}{ Tabla 1. Participantes de la Investigación } \\
\hline Grado & Mujeres & Hombres & Total \\
\hline Séptimo & 135 & 75 & 210 \\
\hline Octavo & 60 & 51 & 111 \\
\hline Noveno & 51 & 35 & 86 \\
\hline Décimo & 62 & 32 & 78 \\
\hline Undécimo & 45 & 33 & 18 \\
\hline Duodécimo & 15 & 3 & 94 \\
\hline Total & 368 & 229 & 597 \\
\hline
\end{tabular}


El 62\% (368) de los participantes son mujeres, frente al 38\% (229) que son hombres. Respecto a su edad, la media se sitúa en 14 años, encontrándose al 62\% de los participantes en el intervalo de edad entre 11-14 años. En referencia a la variable grado académico: el 35\% son del grado séptimo; $19 \%$ son del grado octavo; $14 \%$ del grado noveno; $16 \%$ del décimo grado; $13 \%$ del undécimo grado; y $3 \%$ duodécimo grado. Es importante mencionar que los participantes en su mayoría son del área urbana representada con el $72 \%$, y un $28 \%$ del área rural. Otra característica de la muestra es que la mayoría de los estudiantes utilizan tecnologías de la información y comunicación para acceder a sus estudios virtuales. Para concluir, hay que indicar que el tipo de muestreo que hemos utilizado ha sido el muestreo no probabilístico causal o accidental, que es aquel en el cual el investigador selecciona directa e intencionalmente la muestra, debido fundamentalmente a que tiene fácil acceso a la misma y es representativa de la población (Gil, Rodríguez y García, 1995; Albert, 2006; Sabariego, 2004).

\subsection{Instrumentos de recogida de datos}

El instrumento para la recopilación de la información fue la EAM. Según Palacios et al. (2014) el instrumento está constituido por 32 ítems, aglutinados en 4 dimensiones. Los estudiantes pueden indicar su agrado de acuerdo o desacuerdo con las afirmaciones propuestas por media de una escala Likert de 5 valores. Los factores asociados son: percepción de la incompetencia matemática (12 ítems); gusto por las matemáticas (12 ítems); percepción de utilidad (4 ítems); y autoconcepto matemático (4 ítems). Cabe resaltar que se adaptaron algunos de los ítems del instrumento, ya que por el contexto de los estudiantes se consideró que no iba a ser comprensible.

\begin{tabular}{|c|c|l|l|}
\hline \multicolumn{2}{|c|}{ Factor } & $N^{\circ}$ & \multicolumn{1}{c|}{ Tabla 2. Ítems adaptados por factor } \\
\hline $\begin{array}{l}\text { Percepción de incom- } \\
\text { petencia matemática }\end{array}$ & 4 & $\begin{array}{l}\text { Me siento más torpe en } \\
\text { matemáticas que la mayoría de } \\
\text { mis compañeros por la pregunta. }\end{array}$ & $\begin{array}{l}\text { En matemáticas me siento más torpe } \\
\text { que la mayoría de mis compañeros. }\end{array}$ \\
\cline { 2 - 5 } & 10 & $\begin{array}{l}\text { Salvo en unos pocos casos, por } \\
\text { mucho que me esfuerce no con- } \\
\text { sigo entender las matemáticas. }\end{array}$ & $\begin{array}{l}\text { Por mucho que me esfuerce no con- } \\
\text { sigo entender las matemáticas, salvo } \\
\text { en algunos casos. }\end{array}$ \\
\hline \multirow{2}{*}{$\begin{array}{l}\text { Gustor por las } \\
\text { matemáticas }\end{array}$} & 15 & $\begin{array}{l}\text { Si tuviera oportunidad me apun- } \\
\text { taría a asignaturas optativas. }\end{array}$ & $\begin{array}{l}\text { Si tuviera oportunidad me apuntaría } \\
\text { a asignaturas optativas relacionadas } \\
\text { con matemáticas. }\end{array}$ \\
\cline { 2 - 5 } & 16 & $\begin{array}{l}\text { Puedo pasarme horas estudiando } \\
\text { matemáticas y haciendo. }\end{array}$ & $\begin{array}{l}\text { Puedo pasarme horas estudiando y } \\
\text { practicando matemáticas. }\end{array}$ \\
\cline { 2 - 5 } & 17 & $\begin{array}{l}\text { La materia que se imparte en las } \\
\text { clases de matemáticas es muy } \\
\text { interesante. }\end{array}$ & $\begin{array}{l}\text { El contenido que se imparte en las } \\
\text { clases de matemáticas es muy intere- } \\
\text { sante. }\end{array}$ \\
\hline & 20 & $\begin{array}{l}\text { Las matemáticas son un rollo. } \\
\text { Las matemáticas son yucas (complica- } \\
\text { das). }\end{array}$ \\
\hline
\end{tabular}


Tabla 2. Ítems adaptados por factor

\begin{tabular}{|l|l|l|l|}
\hline \multicolumn{1}{|c|}{ Factor } & $\mathbf{N}^{\circ}$ & \multicolumn{1}{c|}{ Ítem original } & \multicolumn{1}{c|}{ Ítem adaptado } \\
\hline $\begin{array}{l}\text { Percepción por la uti- } \\
\text { lidad }\end{array}$ & 27 & $\begin{array}{l}\text { Las matemáticas deberían estar } \\
\text { presentes únicamente en las car- } \\
\text { reras de ciencias. }\end{array}$ & $\begin{array}{l}\text { Las matemáticas deberían estar pre- } \\
\text { sentes únicamente en las carreras de } \\
\text { ciencias. (ingenierías, tecnologías, } \\
\text { matemáticas, ciencias naturales). }\end{array}$ \\
\hline
\end{tabular}

Es importante resaltar que el instrumento en este estudio alcanzó una confiabilidad alfa de Cronbach del 9o\%.

\subsection{Procedimiento de administración y enfoque ético}

La administración del instrumento se realizó por parte de los autores durante el primer semestre del 2021. Tenía un carácter anónimo y fue complementado por los sujetos participantes en presencia del profesorado. Con anterioridad a la toma de datos, se obtuvo tanto el consentimiento previo, libre e informado de los participantes, así como la autorización de las autoridades educativas.

\subsection{Análisis de datos}

Para satisfacer el objetivo del estudio se procedió al desarrollo de distintos análisis, empleando el paquete estadístico para las ciencias sociales SPSS v.25. Entre ellos, se aplicó un análisis de consistencia interna, un análisis descriptivo de los datos y un análisis de las correlaciones entre las categorías y pruebas paramétricas.

\section{Resultados}

Se muestran los resultados obtenidos con el instrumento y se procedió a analizarlos, encontrando las siguientes

\begin{tabular}{|l|c|c|c|c|c|c|}
\hline \multicolumn{2}{|c|}{ Criterios } & \multicolumn{5}{c|}{ Tabla 3. Percepción de la incompetencia matemática } \\
\cline { 2 - 6 } & $\begin{array}{c}\text { Fuerte- } \\
\text { mente en } \\
\text { desacuerdo }\end{array}$ & $\begin{array}{c}\text { En de- } \\
\text { sacu- } \\
\text { erdo }\end{array}$ & $\begin{array}{c}\text { Indife- } \\
\text { rente }\end{array}$ & $\begin{array}{c}\text { De acu- } \\
\text { erdo }\end{array}$ & $\begin{array}{c}\text { Fuerte- } \\
\text { mente de } \\
\text { acuerdo }\end{array}$ & $\begin{array}{c}\text { Puntuación } \\
\text { Media (SD) }\end{array}$ \\
\hline $\begin{array}{l}\text { 1. En matemáticas me cuesta trabajo de- } \\
\text { cidir qué tengo que hacer. }\end{array}$ & 23 & 28 & 23 & 20 & 6 & \multirow{2}{*}{$(2.58) 1.21$} \\
\hline $\begin{array}{l}\text { 2. Me suelo sentir incapaz de resolver } \\
\text { problemas matemáticos }\end{array}$ & 29 & 26 & 23 & 16 & 5 & $(2.42) 1.22$ \\
\hline $\begin{array}{l}\text { 3. Suelo tener dificultades con las } \\
\text { matemáticas }\end{array}$ & 17 & 27 & 25 & 20 & 11 & $(2.82) 1.24$ \\
\hline $\begin{array}{l}\text { 4. En matemáticas me siento más torpe } \\
\text { que la mayoría de mis compañeros. }\end{array}$ & 41 & 28 & 16 & 9 & 5 & $(2.09) 1.19$ \\
\hline
\end{tabular}




\section{INVESTIGACIÓN EDUCATIVA}

Tabla 3. Percepción de la incompetencia matemática

\begin{tabular}{|c|c|c|c|c|c|c|}
\hline \multirow[b]{2}{*}{ Criterios } & \multicolumn{5}{|c|}{ Respuestas \% } & \multirow[b]{2}{*}{$\begin{array}{l}\text { Puntuación } \\
\text { Media (SD) }\end{array}$} \\
\hline & $\begin{array}{c}\text { Fuerte- } \\
\text { mente en } \\
\text { desacuerdo }\end{array}$ & $\begin{array}{l}\text { En de- } \\
\text { sacu- } \\
\text { erdo }\end{array}$ & $\begin{array}{l}\text { Indife- } \\
\text { rente }\end{array}$ & $\begin{array}{l}\text { De acu- } \\
\text { erdo }\end{array}$ & $\begin{array}{l}\text { Fuerte- } \\
\text { mente de } \\
\text { acuerdo }\end{array}$ & \\
\hline 5. Las matemáticas me confunden. & 24 & 23 & 25 & 19 & 9 & $(2.65) 1.27$ \\
\hline $\begin{array}{l}\text { 6. Siempre he tenido problemas con las } \\
\text { matemáticas. }\end{array}$ & 31 & 27 & 22 & 12 & 8 & $(2.39) 1.25$ \\
\hline $\begin{array}{l}\text { 7. Haga lo que haga, siempre saco notas } \\
\text { bajas en matemáticas. }\end{array}$ & 37 & 26 & 21 & 9 & 6 & $(2.21) 1.22$ \\
\hline $\begin{array}{l}\text { 8. En matemáticas me quedo con la mente } \\
\text { en blanco con frecuencia. }\end{array}$ & 34 & 27 & 19 & 14 & 6 & $(2.29) 1.23$ \\
\hline 9. No sé estudiar matemáticas. & 40 & 30 & 18 & 8 & 4 & $(2.08) 1.14$ \\
\hline $\begin{array}{l}\text { 10. Por mucho que me esfuerce no consigo } \\
\text { entender las matemáticas, salvo en algu- } \\
\text { nos casos. }\end{array}$ & 32 & 28 & 21 & 12 & 7 & $(2.33) 1.23$ \\
\hline $\begin{array}{l}\text { 11. Me será siempre difícil aprender } \\
\text { matemáticas. }\end{array}$ & 40 & 29 & 17 & 9 & 5 & $(2.11) 1.17$ \\
\hline $\begin{array}{l}\text { 12. Soy una de esas personas que no nació } \\
\text { para aprender matemáticas. }\end{array}$ & 38 & 23 & 22 & 8 & 9 & $(2.29) 1.3$ \\
\hline \multicolumn{7}{|c|}{ Puntuación Total a } \\
\hline Mediana (rango) & \multicolumn{6}{|l|}{$48(12-60)$} \\
\hline Media (SD) & \multicolumn{6}{|l|}{$28.25(11.45)$} \\
\hline Varianza & \multicolumn{6}{|l|}{131.00} \\
\hline Puntuación total \% & \multicolumn{6}{|l|}{$47 \%$} \\
\hline
\end{tabular}

a Máxima puntuación $=60$

$\mathrm{Al}$ analizar el factor de la incompetencia matemática, se puede observar que la media obtenida (28.25) con respecto a la media teórica (36), que los estudiantes en su mayoría no creen que sean incompetentes en matemáticas, es decir, que cierta seguridad sobre la adquisición y utilización de competencias matemáticas. Sin embargo, un 31\% de los estudiantes considera que suele tener dificultades con las matemáticas, mientras que un $70 \%$, considera que sabe cómo estudiar matemáticas.

\begin{tabular}{|c|c|c|c|c|c|c|}
\hline \multirow[b]{2}{*}{ Criterios } & \multicolumn{5}{|c|}{ Respuestas \% } & \multirow[b]{2}{*}{$\begin{array}{l}\text { Puntuación } \\
\text { Media (SD) }\end{array}$} \\
\hline & $\begin{array}{l}\text { Fuerte- } \\
\text { mente en } \\
\text { desacu- } \\
\text { erdo }\end{array}$ & $\begin{array}{c}\text { En } \\
\text { desacu- } \\
\text { erdo }\end{array}$ & $\begin{array}{l}\text { Indife- } \\
\text { rente }\end{array}$ & $\begin{array}{l}\text { De acu- } \\
\text { erdo }\end{array}$ & $\begin{array}{l}\text { Fuerte- } \\
\text { mente de } \\
\text { acuerdo }\end{array}$ & \\
\hline $\begin{array}{l}\text { 13. Me resulta divertido estudiar } \\
\text { matemáticas. }\end{array}$ & 19 & 23 & 28 & 19 & 11 & $(2.8) 1.26$ \\
\hline $\begin{array}{l}\text { 14. Cuando tengo que estudiar matemáti- } \\
\text { cas voy a la tarea con cierta alegría. }\end{array}$ & 21 & 23 & 29 & 17 & 9 & (2.7)1.24 \\
\hline
\end{tabular}


Actitudes hacia las matemáticas en estudiantes de Honduras

\begin{tabular}{|c|c|c|c|c|c|c|}
\hline $\begin{array}{l}\text { 15. Si tuviera oportunidad me apuntaría } \\
\text { a asignaturas optativas relacionadas con } \\
\text { matemáticas. }\end{array}$ & 24 & 27 & 26 & 15 & 8 & $(2.57) 1.23$ \\
\hline $\begin{array}{l}\text { 16. Puedo pasarme horas estudiando y } \\
\text { practicando matemáticas }\end{array}$ & 25 & 24 & 24 & 17 & 10 & $(2.62) 1.28$ \\
\hline $\begin{array}{l}\text { 17. El contenido que se imparte en las } \\
\text { clases de matemáticas es muy interesante. }\end{array}$ & 10 & 18 & 29 & 27 & 16 & $(3.2) 1.2$ \\
\hline $\begin{array}{l}\text { 18. Las matemáticas son una de las asigna- } \\
\text { turas más aburridas. }\end{array}$ & 33 & 27 & 26 & 7 & 6 & $(2.25) 1.16$ \\
\hline 19. Me gustan las matemáticas & 17 & 22 & 27 & 21 & 13 & $(2.92) 1.28$ \\
\hline $\begin{array}{l}\text { 20. Las matemáticas son yucas (complica- } \\
\text { das) }\end{array}$ & 18 & 20 & 32 & 18 & 11 & $(2.84) 1.25$ \\
\hline $\begin{array}{l}\text { 21. Me siento cómodo resolviendo prob- } \\
\text { lemas matemáticos. }\end{array}$ & 18 & 22 & 31 & 18 & 11 & $(2.83) 1.23$ \\
\hline $\begin{array}{l}\text { 22. Toca clase de matemáticas ¡Qué hor- } \\
\text { ror! }\end{array}$ & 37 & 29 & 20 & 9 & 5 & $(2.17) 1.17$ \\
\hline $\begin{array}{l}\text { 23. No soporto estudiar matemáticas, in- } \\
\text { cluso las partes más fáciles. }\end{array}$ & 40 & 29 & 20 & 6 & 4 & $(2.04) 1.09$ \\
\hline 24.Las matemáticas son fáciles. & 23 & 23 & 33 & 11 & 9 & $(2.58) 1.21$ \\
\hline \multicolumn{7}{|l|}{ Puntuación Total ${ }^{a}$} \\
\hline Mediana (rango) & \multicolumn{6}{|l|}{$48(12-60)$} \\
\hline Media (SD) & \multicolumn{6}{|l|}{$31.53(8.84)$} \\
\hline Varianza & \multicolumn{6}{|l|}{7.152} \\
\hline Puntuación total \% & \multicolumn{6}{|l|}{$53 \%$} \\
\hline
\end{tabular}

a Máxima puntuación $=60$

Con respecto al factor gusto por las matemáticas el cual está conformado por 12 ítems, que miden el gusto o disfrute al realizar actividades o trabajos matemáticos. Comparando la media obtenida (31.53) con respecto a la media teórica (36) se puede concluir que la mayoría de estudiantes se inclinan a no tener gusto por las matemáticas. En este mismo sentido, un $69 \%$ de los estudiantes consideran que no soportan estudiar matemáticas, sin embargo, un $43 \%$ consideran que la clase de matemáticas es muy interesante.

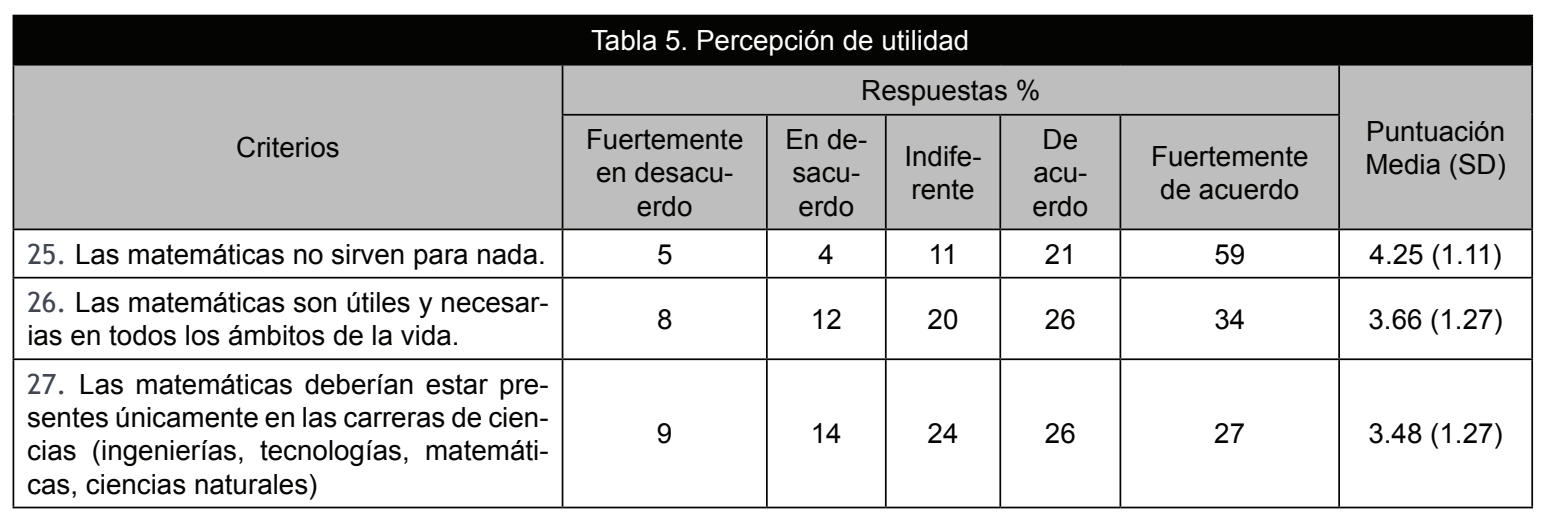




\section{INVESTIGACIÓN EDUCATIVA}

\begin{tabular}{|l|l|l|l|l|l|l|}
\hline $\begin{array}{l}\text { 28. Aprender matemática es cosa de } \\
\text { unos pocos. }\end{array}$ & 9 & 13 & 25 & 28 & 25 & $3.64(1.24)$ \\
\hline Puntuación Total ${ }^{a}$ & $16(4-20)$ \\
\hline Mediana (rango) & $14.87(2.67)$ \\
\hline Media (SD) & 7.15 \\
\hline Varianza & $74 \%$ \\
\hline Puntuación total \% & á́xima puntuación $=20$
\end{tabular}

Los resultados sobre la utilidad referente al valor, creencias o percepciones que los estudiantes consideren sobre la utilidad que les dan a las matemáticas en la vida cotidiana, llaman bastante la atención, ya que es el factor en el que su media (14.87), está más alejada con respecto a media teórica (12), mostrando que más de la mitad de los estudiantes perciben como útiles las matemáticas. Además, se detalla que, el $80 \%$ de los estudiantes están fuertemente en desacuerdo en que las matemáticas no sirven para nada, en contraste con el $23 \%$ que consideran que las matemáticas solo deberían estar presentes en carreras relacionadas con las ciencias.

\begin{tabular}{|c|c|c|c|c|c|c|}
\hline \multirow[b]{2}{*}{ Criterios } & \multicolumn{5}{|c|}{ Respuestas \% } & \multirow[b]{2}{*}{$\begin{array}{l}\text { Puntuación } \\
\text { Media (SD) }\end{array}$} \\
\hline & $\begin{array}{l}\text { Fuerte- } \\
\text { mente en } \\
\text { desacuerdo }\end{array}$ & $\begin{array}{l}\text { En } \\
\text { desacu- } \\
\text { erdo }\end{array}$ & $\begin{array}{l}\text { Indife- } \\
\text { rente }\end{array}$ & $\begin{array}{l}\text { De acu- } \\
\text { erdo }\end{array}$ & $\begin{array}{l}\text { Fuerte- } \\
\text { mente de } \\
\text { acuerdo }\end{array}$ & \\
\hline $\begin{array}{l}\text { 29. Puedo llegar a ser un buen estudiante de } \\
\text { matemáticas. }\end{array}$ & 10 & 14 & 26 & 27 & 23 & $(3.4) 1.25$ \\
\hline $\begin{array}{l}\text { 30. Si me lo propusiera creo que llegaría a dom- } \\
\text { inar bien las matemáticas. }\end{array}$ & 8 & 13 & 23 & 28 & 28 & $(3.54) 1.24$ \\
\hline 31. Se me da bien calcular mentalmente. & 14 & 25 & 33 & 19 & 9 & $(2.84) 1.16$ \\
\hline $\begin{array}{l}\text { 32. Para mi maestros y profesores de matemáti- } \\
\text { cas soy un buen estudiante. }\end{array}$ & 14 & 22 & 35 & 20 & 9 & $(2.9) 1.16$ \\
\hline \multicolumn{7}{|l|}{ Puntuación Total ${ }^{a}$} \\
\hline Mediana (rango) & \multicolumn{6}{|l|}{$16(4-20)$} \\
\hline Media (SD) & \multicolumn{6}{|l|}{$12.68(3.94)$} \\
\hline Varianza & \multicolumn{6}{|l|}{15.52} \\
\hline Puntuación total \% & \multicolumn{6}{|l|}{$63 \%$} \\
\hline
\end{tabular}

Finalmente, al medir la autoimagen que tiene el estudiante sobre las matemáticas y cómo las percibe que corresponde al factor del autoconcepto, se encontró que su media (12.68) está por encima de la media teórica (12), por lo que se deduce que en su mayoría los estudiantes tienen un buen concepto matemático sobre sí mismos. Se infiere también, que un 56\% de los estudiantes consideran que si se lo propusieran podrían llegar a dominar las matemáticas, pero un $39 \%$ consideran que se les dificulta calcular mentalmente. 
Tabla 7. Análisis de correlaciones entre las categorías ( $r$-Pearson)

\begin{tabular}{|l|c|c|c|c|}
\hline \multicolumn{1}{|c|}{ Factores } & Categoría 1 & Categoría 2 & Categoría 3 & Categoría 4 \\
\hline $\begin{array}{l}\text { 1. Percepción de la incompe- } \\
\text { tencia matemática }\end{array}$ & 1 & -0.342 & 0.187 & -0.072 \\
\hline 2. Gusto por las matemáticas & & 1 & 0.002 & 0.158 \\
\hline 3. Percepción de utilidad & & & 1 & 0.601 \\
\hline 4. Autoconcepto matemático & & & & 1 \\
\hline
\end{tabular}

En la tabla 7, se puede observar las correlaciones entre los factores actitudinales, se puede decir que la percepción de la incompetencia matemática es inversamente proporcional al gusto por las matemáticas y es una correlación negativa entre débil y media. También, se puede observar que la percepción de utilidad y el autoconcepto matemático poseen una correlación entre 0.5 y 0.75 por lo que tiene una correlación positiva entre media y considerable. Se puede observar además que hay una pequeña correlación entre el gusto por las matemáticas y la percepción de incompetencia, esto puede ser debido a que los estudiantes consideran que no les gustan las matemáticas. Finalmente, se puede observar una tendencia inversamente proporcional, aunque muy baja, entre la percepción de incompetencia y el autoconcepto matemático, por lo que entre mayor sea la sensación de incompetencia, menor será la percepción de utilidad.

Al relacionar los otros factores entre sí, no muestran una correlación mayor a débil tanto positiva como negativa.

\begin{tabular}{|l|c|c|c|c|}
\hline \multirow{2}{*}{\multicolumn{1}{|c}{ Factores }} & Género & Edad & Grado Académico & Zona de residencia \\
\cline { 2 - 5 } & $p$ & $p$ & $p$ & $p$ \\
\hline $\begin{array}{l}\text { Percepción de la incompeten- } \\
\text { cia matemática }\end{array}$ & 0.01 & 0.00 & 0.01 & 0.01 \\
\hline Gusto por las matemáticas & 0.93 & 0.66 & 0.52 & 0.15 \\
\hline Percepción de utilidad & 0.50 & 0.70 & 0.50 & 0.70 \\
\hline Autoconcepto matemático & 0.89 & 0.58 & 0.50 & 0.05 \\
\hline
\end{tabular}

Al realizar las pruebas no paramétricas sobre cada uno de los factores en relación con el género, edad, grado académico y zona de residencia, para determinar si hay diferencias significativas dentro de cada uno de los grupos, se puede observar que, en los factores de gusto por las matemáticas, percepción de utilidad y autoconcepto matemático, no hay diferencias significativas al comparar los géneros, las edades, el grado académico o la zona de residencia. Al contrario, el factor de reducción de incompetencia muestra que hay diferencias significativas en cada uno de estos grupos ya que el valor $\mathrm{p}<0.05$. Con respecto al género se observó que las mujeres tenían una percepción de incompetencia más alta que la de los varones. En relación a la edad los estudiantes con edades de 11 hasta 16, se comportan con una percepción de 
incompetencia directamente proporcional a su edad, es decir que a mayor edad mayor sensación de incompetencia matemática, sin embargo, este patrón de comportamiento se rompe a partir de los 17 años. En relación al grado de los estudiantes se mostró que los estudiantes a medida avanzaban en los grados se sentían más incompetentes matemáticamente. Finalmente, con respecto a la zona de residencia se mostró que los estudiantes que habitan en zonas urbanas tienen una menor percepción de incompetencia en relación a los de la zona rural.

\section{Discusión y conclusiones}

En este estudio se analizaron las actitudes hacia la matemática, es decir el componente afectivo mediante cuatro factores y relacionándolos con el género, edad, grado académico y zona de residencia. Como se ha mencionado anteriormente las actitudes de las matemáticas juegan un papel importante hacia la enseñanza y aprendizaje de las matemáticas, tanto así que estudios han mostrado que el rendimiento académico es directamente proporcional con estas actitudes, es por ello que los docentes de matemáticas deben efectuar acciones inmediatas que generen un impacto positivo en las actitudes hacia las matemáticas.

Al analizar cada uno de los factores se ha encontrado de forma general que los estudiantes tienen una actitud positiva hacia las matemáticas en los factores percepción de incompetencia matemática, percepción de utilidad y autoconcepto matemático, al contrario, con el factor gusto por las matemáticas en el cual se ha observado una actitud negativa al respecto.

Con respecto a las relaciones entre los diferentes factores se encontró que la percepción de incompetencia es inversamente proporcional al gusto por las matemáticas con un factor de corrección de -0.342, es decir que si se aumenta el gusto por las matemáticas disminuirá la sensación de ser incompetentes en la materia. Además de estos, los factores percepción de utilidad y autoconcepto matemático mostraron poseer una proporcionalidad directa.

En cuanto a la a existencia de diferencias significativas entre los datos demográficos se ha observado que no hay diferencias significativas con respecto a los factores de autoconcepto matemático, gusto por las matemáticas y percepción de utilidad, coincidiendo con los resultados de las investigaciones de Zarrazaga (2006) y Muñoz y Mato (2008).

Dentro del factor percepción de incompetencia matemática se encontraron diferencias significativas en todas las variables demográficas analizadas. En relación con el grado académico se determinó que entre más elevado es el grado académico de los estudiantes, las actitudes negativas hacia las matemáticas también se elevan, 
resultados similares a los de Zarrazaga (2006). Con respecto al género se observó que los hombres tienen una menor percepción de incompetencia matemática con relación a las mujeres que se perciben con mayor competencia matemática.

En cuanto a la relación entre la zona de residencia versus las actitudes hacia las matemáticas, se demuestra que los estudiantes que viven en la zona urbana tienen una menor percepción de incompetencia matemática, en contraste con los que residen en las áreas rurales de Honduras. Debido a que este estudio se realizó en tiempos de pandemia Covid-19 se percibe que en su mayoría se puede ser debido al acceso a internet de buena calidad y a la destreza para manejar las TIC, ya que en las zonas rurales de Honduras la calidad del servicio de internet QoS es de mala calidad, como también los fallos en el fluido eléctrico están a la orden del día, por lo cual se recomienda que para estudios futuros tomar en cuenta el acceso a internet y la destreza para manejar equipo tecnológico.

\section{Lista de referencias}

Aiken Jr, L. R., \& Dreger, R. M. (1961). The effect of attitudes on performance in mathematics. Journal of Educational psychology, 52(1), 19

Aiken, L. (1974). Two Scales of Attitude toward Mathematics. Journal for Research in Mathematics Education, 5(2), 67-71. doi:10.2307/748616

Albert, M. (2006). La investigación educativa. Claves teóricas. Madrid: MCGraw-Hill.

Auzmendi, E. (1992). Las actitudes hacia la matemática-estadística en las enseñanzas media y universitaria. Bilbao, España: Ediciones Mensajero.

Bisquerra, R., \& Alzina, R. B. (2012). Metodología de la investigación educativa. Editorial La Muralla.

Cantero, J. M. M., \& Vázquez, M. D. M. (2008). Análisis de las actitudes respecto a las matemáticas en alumnos de ESO. Revista de Investigación Educativa, 26(1), 209-226. Recuperado de: https://www.redalyc.org/pdf/2833/283321884011.pdf

Fennema, E., y Sherman, J. (1976). Fennema-Sherman Mathematics Attitudes Scales: Instruments Designed to Measure Attitudes toward the Learning of Mathematics by Females and Males. Journal for Research in Mathematics Education, 7(5), 324-326. doi:10.2307/748467

Flores, W., \& Auzmendi, E. (2018). Actitudes hacia las matemáticas en la enseñanza universitaria y su relación con las variables género y etnia. Profesorado, 
revista de currículum y formación del profesorado, 22(3), 231-248. https:// doi.org/10.30827/profesorado.v22i3.8000

Gil, J., Rodríguez, G., y García, E. (1995). Estadística básica aplicada a las Ciencias de la Educación. Sevilla: Kronos.

Gómez-Chacón, I. (1997). Procesos de aprendizaje en matemáticas con poblaciones de fracaso escolar en contextos de exclusión social. Las influencias afectivas en el conocimiento de las matemáticas. Tesis doctoral. Universidad Complutense. Recuperado de: https://eprints.ucm.es/id/eprint/2249/1/T22147.pdf

Gómez-Chacón, I. (2000). Matemática emocional. Los afectos en el aprendizaje matemático. Madrid: Narcea. Recuperado de: https://revistas.unav.edu/index.php/ estudios-sobre-educacion/article/view/27381/22846

Hernández, R., Baptista Lucio, P., \& Fernández Collado, C. (2014). Metodología de la Investigación. Editorial McGraw Hill.

Martínez, O. (2008). Actitudes hacia la matemática. Sapiens. Revista Universitaria de Investigación, 9 (1), 237-256. 1317-5815. Recuperado de: https://www.redalyc. org/articulo.oa?id $=41011135012$

Morales, L., Sánchez, J., Ortega, G. y García O. (2013). Actitud hacia las matemáticas: Un estudio comparativo entre estudiantes panameños y mexicanos. Recuperado de: https://www.researchgate.net/publication/316702089_Actitud_hacia_la_ Matematica_Un_estudio_comparativo_entre_estudiantes_panamenos_y_ mexicanos

Muñoz, J. y Mato, M. (2008). Análisis de las actitudes respecto a las matemáticas en alumnos de ESO. Revista de Investigación Educativa, 26(1), 209-226. Recuperado de: https://www.redalyc.org/articulo.oa?id=283321884011

Nortes, R. y Nortes, A. (2017). Agrado y utilidad de las matemáticas en la formación inicial de maestros de educación primaria. PNA, 12(1), 27-44. Recuperado de: https://revistaseug.ugr.es/index.php/pna/article/view/6537/5667

Palacios, A., Arias, V., \& Arias, B. (2014). Las actitudes hacia las matemáticas: construcción y validación de un instrumento para su medida. Revista de psicodidáctica, 19(1), 67-91.

Sabariego, M. (2004). El proceso de investigación. En R. Bisquerra, Metodología de la investigación educativa (127-163). Madrid: La Muralla. 
Zarrazaga, A. (2006). La actitud hacia las matemáticas y el rendimiento académico. Revista Memorias, 7(1), 53-62. Recuperado de: http://memorias.um.edu.mx/ ojs/index.php/rev/article/view/23 